\title{
DETERMINANTES DE LA ESTRUCTURA DE CAPITAL EN LA INDUSTRIA QUÍMICA COLOMBIANA
}

\section{Determinants of the Capital Structure in the Colombian Chemical Industry}

Cristián Andrés Echeverri Castaño, lavier Esteban

Escobar Ortega y Judith Vergara Garavito 


\section{Determinants of the Capital Structure in the Colombian Chemical Industry}

Cristián Andrés Echeverri Castaño(a), Javier Esteban Escobar Ortega(b) y Judith Vergara Garavito(c)

Palabras clave: Estructura de capital, Industria química, Apalancamiento.

Keywords: Capital structure, Chemical Industry, Leverage.

Clasificación JEL: G32, L93

Received: 11/06/2020

Accepted: $14 / 04 / 2021$

Published: 01/06/2021

\section{Resumen}

El objetivo de este estudio fue encontrar los determinantes de la estructura de capital en las empresas del sector químico colombiano. Para ello se tomaron datos anuales de 233 firmas en el período comprendido entre 2009 y 2018, lo que permitió construir un panel desbalanceado con 1980 observaciones. Los resultados demuestran que la rentabilidad, el crecimiento, el tamaño y la liquidez son determinantes en la decisión de estructura de capital de las firmas del sector químico en Colombia.

\section{Abstract}

This research aimed to find the determinants of the capital structure of Colombian companies in the chemical sector. For that, annual data was taken from 233 firms in the period ranging from 2009 to 2018 , which allowed to build an unbalanced panel with 1980 observations. The results showed that profitability, growth, size, and liquidity are decisive in the decision of capital structure of firms in the chemical sector in Colombia.
Iavier Esteban Escobar Ortega. Ingeniero

Financiera. Gerente del área de analítica de negocio en Banistmo empresa del Grupo

Bancolombia-Panamá. jeo_528@hotmail.com

c. Judith Vergara Garavito. Ph.D. Profesora. Coordinadora Maestría en Administración Financiera. Departamento de Finanzas.

Escuela de Economía y Finanzas. Universidad EAFIT. jvergar8@eafit.edu.co 


\section{Introducción}

El sector químico tiene una de las industrias manufactureras más grandes del mundo. La industria en general juega un papel importante en el desarrollo de la economía del mundo y es el mayor consumidor de recursos naturales no renovables, así como uno de los mayores contaminantes globales (Aristizábal, 2018). Desde mediados de los años ochenta, la industria química global creció cada año en un $7 \%$ hasta 2010. Para el año 2030, se proyecta que Asia ocupará el $66 \%$ del mercado, mientras que Europa y el Nafta reducirán su participación a un 15 y un $12 \%$, respectivamente (Montes, 2015). $\mathrm{El}$ alto crecimiento en las ventas de esta industria, tanto el histórico como el proyectado, genera en ella transformaciones importantes en la estructura de capital, pues existe una demanda permanente de recursos para financiar sus actividades.

Las combinaciones posibles que se pueden encontrar en la estructura de capital varían entre regiones y sectores de la economía. Lo anterior condujo a que algunos teóricos desarrollaran hipótesis para intentar explicar el fenómeno. Modigliani y Miller (1958) demostraron que la diferencia que existe entre el costo de los recursos del patrimonio y el de los de la deuda es significativamente pequeña, por lo que no tiene diferencia financiarse con una fuente u otra. En su trabajo seminal, estos autores demostraron que el valor de mercado de la firma está determinado por su capacidad de generar ganancias y el riesgo de los activos subyacentes. Posteriormente, surgieron otras teorías que buscaban indagar acerca de las decisiones de estructura de capital de la firma, tales como la teoría de la compensación estática o trade-off (STT) (Myers, 1977), la teoría del orden jerárquico o pecking order (POT) (Myers, 1984) y la de la señalización (Ross, 1977).

A nivel latinoamericano, se encuentra el estudio realizado por Céspedes, González y Molina (2010), que provee evidencia empírica a hallazgos previos de que el tamaño, las oportunidades de crecimiento, la tangibilidad de los activos, la rentabilidad y los impuestos son relevantes para explicar el apalancamiento en la muestra de empresas latinoamericanas. Para el caso del mercado mexicano, el estudio de Hernández, Ríos y Garrido (2015) concluye que empresas exportadoras y de gran tamaño, al resolver problemas de información asimétrica con sus acreedores, logran continuar financiándose con sus acreedores a pesar de la crisis.

Por su parte, en el contexto colombiano, se han desarrollado estudios sobre determinantes de estructura de capital para algunos sectores o industrias. Tal es el caso del estudio desarrollado por Pérez (2005), quien de manera analítica explora los determinantes de la estructura de capital de las grandes empresas del sector de alimentos en Colombia. Así mismo, Virgen y Rivera (2012) muestran que, en empresas del subsector droguería-cosméticos y hogar-aseo, también en Colombia, la rentabilidad y el costo de la deuda son determinantes de la reducción de los niveles de endeudamiento, pese a que las variables influyentes de la estructura financiera de las empresas que conforman un subsector difieren de manera importante del otro subsector. Recientemente se encontró la investigación de Quintero y Rueda (2018), en la que se analizaron los factores en los que se basan los administradores financieros para tomar decisiones sobre el endeudamiento para compañías que cotizan en bolsa. Es de aclarar que las diferencias entre los sectores estudiados, los períodos tomados para la respectiva muestra y las variables incluidas en cada estudio imposibilitan generalizar los resultados obtenidos en la industria química nacional. En la revisión de literatura efectuada, se encontraron pocos estudios sobre estructura de capital de la industria química, por lo que no hay suficiente evidencia empírica que permita conocer cuáles son las variables determinantes del apalancamiento en este sector. 
Debido a lo anterior, se hace necesario encontrar los principales determinantes de la estructura de capital que afectan la industria química colombiana para generar una fuente de información que ayude a los gerentes de compañías a tomar decisiones que maximicen el valor de las firmas colombianas de este sector. Es así como este trabajo contribuye al cuerpo teórico de las finanzas corporativas, en particular a la literatura de la estructura de capital de empresas pertenecientes a la industria química en un país emergente como Colombia. El artículo está organizado de la siguiente manera: en la segunda sección se presenta el marco teórico, en el que se hace un recorrido por los conceptos fundamentales sobre las teorías de estructura de capital y se analiza la industria para entender sus riesgos. En la tercera sección se presenta la metodología, en la que se define el tamaño de la muestra, las variables, las hipótesis y se detalla la técnica analítica utilizada para probar las hipótesis planteadas. En la cuarta sección se presentan los resultados y hallazgos del modelo. Por último, en la quinta sección se presentan las conclusiones, las implicaciones prácticas y teóricas, así como las recomendaciones para futuras investigaciones.

\section{Marco teórico}

Durante el siglo XX, empezaron a aparecer teorías que intentaban explicar la estructura de capital de las empresas y que buscaban identificar la composición óptima que maximizaba su valor. En dicho momento, de acuerdo con un enfoque de mercados perfectos, apareció la tesis tradicional propuesta por Durand (1952) y la tesis de irrelevancia de Modigliani y Miller (1958). Durand (1952) aseguró que el uso moderado de la deuda incrementa el valor de la compañía siempre y cuando el costo de capital del patrimonio sea menor que el beneficio de incrementar el apalancamiento. En su estudio también analizó que maximizar los ingresos no siempre logra generar un mayor valor de la compañía, por lo que los directivos deben asegurarse de maximizar el valor presente de sus flujos futuros y no el de los ingresos actuales. Más adelante, Modigliani y Miller (1958) propusieron que, bajo el supuesto de mercados perfectos, el valor de las empresas no se ve afectado por la estructura de capital. Es decir, no existen imperfecciones de mercado tales como los impuestos, los costos de transacción y la asimetría de información, entre otras; esto hace irrelevante las decisiones de estructura financiera. Su postulado estaba basado en dos proposiciones: el valor de mercado de la empresa y el costo de capital. La primera expone que el valor de mercado de una empresa y el costo de capital son independientes de su estructura de capital, es decir, que el valor de la empresa no depende de su financiación. La segunda postula que el valor de la empresa está dado por el potencial que tiene para generar ganancias y el riesgo de los activos que posee, sin determinar la forma en que son financiados.

Más tarde, en el marco de la hipótesis de mercados imperfectos, como ya se mencionó, se desarrollaron las principales teorías modernas: la STT de Myers (1977), la de los costos de agencia de Jensen y Meckling (1976) y la POT de Myers (1984).

\subsection{Teoría de equilibrio estático o static trade-off theory (STT)}

Basada en la investigación sobre impuestos de Modigliani y Miller (1963), en los estudios de costos de bancarrota y dificultades financieras de Warner (1977) y en las ideas de la literatura de la agencia de Jensen y Meckling (1976), la STT sugiere que las empresas tienen una estructura de capital óptima única que se equilibra entre la ventaja fiscal de la financiación de la deuda (es decir, escudos fiscales de la deuda) y los costos de las dificultades financieras. La STT establece que una empresa sigue un objetivo en la relación entre deuda y capital, por tanto, los beneficios y los costos vinculados con la opción de endeudamiento establecen este índice objetivo. Así mismo, considera que los impuestos, 
los costos de dificultades financieras y los costos de agencia determinan una estructura de capital óptima. La estructura de capital óptima es una combinación de fuentes de financiamiento que genera el mayor precio de mercado.

\subsection{Teoría del orden jerárquico o pecking order theory (POT)}

La POT fue presentada por Myers (1984). Postula que las empresas siguen una jerarquía financiera de decisiones al establecer su estructura de capital y se estudia con base en la información asimétrica entre gerentes e inversores. Los gerentes tienen más información sobre el verdadero valor de la firma y el riesgo empresarial en comparación con los inversores externos. Según Myers (1984), las organizaciones financian sus actividades con utilidades retenidas siempre que sea posible. Si las utilidades retenidas no fueran un recurso suficiente o adecuado, se utilizaría la deuda. Solo en casos extremos, las compañías emplearían nuevos aportes de patrimonio. Por lo tanto, el orden de las fuentes de financiamiento sería 1) utilidades retenidas, 2) valores de corto plazo, 3) deuda, 4) acciones preferentes y 5) como última alternativa de financiamiento, acciones comunes.

La información asimétrica entre la administración y los nuevos titulares es la razón por la que las empresas son reacias a emitir capital. Myers y Majluf (1984) señalaron que la subvaloración sería el resultado de una menor información en poder de los inversionistas potenciales con respecto a la administración, en lo referente a los flujos de efectivo esperados de los activos de la compañía, tanto actuales como futuros. Si se tienen en cuenta estas asimetrías de información, los inversores inferirían que la administración emite acciones solo cuando fuese demasiado caro. Por lo tanto, el nuevo patrimonio emitido podría venderse con un descuento, lo que se consideraría como una transferencia de riqueza de los inversores existentes a los nuevos. Este problema podría evitarse si las empresas utilizaran recursos generados internamente, como, por ejemplo, las ganancias retenidas.

\subsection{Teoría de la señalización}

Spence (1973) desarrolló una teoría de señalización que plantea que una buena empresa puede distinguirse de una mala porque envía una señal creíble sobre su calidad a los mercados de capitales. La señal será creíble solo si la mala compañía no puede imitar a la buena con el envío de la misma señal. Si el costo de la señal es más alto para la mala que para la buena, la mala no imitaría la señal, y, por tanto, sería creíble.

Ross (1977) mostró cómo la deuda podría usarse como una señal costosa para separar las empresas buenas de las malas. Según la información asimétrica entre la administración y los inversores, las señales de las compañías son cruciales para obtener recursos financieros. Ross supuso que los gerentes (los expertos) conocen la verdadera distribución de los rendimientos de las empresas, pero los inversores no. La señalización de una mayor deuda por parte de los gerentes sugiere un futuro optimista y las compañías de alta calidad usarían más deuda mientras que las de baja calidad tendrían niveles de deuda más bajos.

2.4 Determinantes de la estructura de capital en la industria química en el mundo

Este ha sido un tema de particular interés entre académicos y practitioners. Recientemente, $\underline{\text { Cintra, }}$ Selan, Albanez y Do Valle (2012) analizaron la estructura de capital y las características financieras de la industria química brasileña en el período comprendido entre 1999 y 2008. Con una metodología 
empírica-analítica clasificaron las empresas de la industria según su moneda (nacional o extranjera) y sus características. Los resultados de esta investigación concluyeron que, primero, las fuentes nacionales y extranjeras de financiamiento contribuyeron de manera significativa al apalancamiento de las compañías químicas brasileñas. Segundo, las variables tamaño, tangibilidad de los activos, intangibilidad, rentabilidad y riesgo también fueron importantes para explicar el nivel de endeudamiento de las firmas analizadas. No obstante, al utilizar solamente 12 empresas como muestra de estudio, los resultados no pueden ser generalizables a toda la población.

Por otro lado, Camara-Turull, Fernández-Izquierdo y Sorrosal-Forradellas (2017) analizaron la estructura de capital de la industria química española durante el período comprendido entre 1999 y 2013 con un doble objetivo. Primero, determinar si los supuestos de la POT se cumplían a lo largo del marco temporal del estudio y, segundo, mostrar cómo la crisis económica ha afectado la estructura de capital de las empresas incluidas en su muestra. Los resultados del estudio arrojaron que las compañías se agruparon en diferentes grados de cumplimiento de la POT. Así mismo, la hipótesis sobre el riesgo experimentó una mayor variación en los períodos anterior y posterior a la crisis.

Por su parte, Bodla y Singh (2017) examinaron los factores que influyen en la estructura de capital de las empresas que pertenecen a las industrias farmacéutica y química, listadas en Mumbai Stock Exchange Ltd. de India. Con base en la información del período 1999-2015, estos autores probaron que sí existe una relación significativa entre el apalancamiento financiero y las variables independientes que se tomaron de la literatura clásica. Los resultados mostraron, además, que el costo de la deuda, el costo del patrimonio, la liquidez y el servicio de la deuda fueron variables significativas para determinar la estructura de capital. Sin embargo, los autores consideran solamente dos subsectores de los que componen el sector químico, y dejan por fuera un importante número de empresas constitutivas de este sector.

Zelgalve y Bērzkalne (2011) analizaron la estructura de capital, evaluaron las posibilidades de obtener capital e identificaron los aspectos de las teorías de estructura de capital en las empresas químicas de Letonia; también analizaron y extrajeron conclusiones sobre la estrategia de financiación de las compañías. Como resultado de su análisis, encontraron que el $80 \%$ de las empresas cumplió la teoría del tiempo del mercado; en el $10 \%$ no fue posible establecer un cumplimiento preciso, debido a la falta de datos; y en el 10 \% restante encontraron rasgos de la POT. La correlación entre el índice de deuda de Letonia (todas las empresas) y el Producto Interno Bruto (PIB) fue significativamente mayor que la encontrada entre el índice de deuda de las empresas químicas y el PIB. La misma conclusión se puede referir al coeficiente de endeudamiento y al monto de los préstamos emitidos. La mayor correlación encontrada se presentó con la cantidad de activos fijos: o,88. Todas las correlaciones fueron positivas: junto con el crecimiento del PIB per cápita, el monto de los préstamos emitidos y el monto de los activos fijos. Sin embargo, la investigación no permite generalizar los hallazgos debido a la falta de datos referida por los autores y al número limitado de empresas.

Tomando como muestra la industria química de Pakistán, Muhammad, Asif y Muhammad $\underline{(2008)}$ intentaron determinar la estructura de capital de estas empresas. El estudio encontró que, al analizar la estructura de capital de una industria específica, se podían determinar atributos únicos, que por lo general no fueron evidentes en el análisis combinado de muchos sectores. Se emplearon seis regresores: 1) el tamaño de la empresa, 2) la tangibilidad de los activos, 3) la rentabilidad, 4) la variación de los ingresos, 5) el escudo fiscal sin deuda (NDTS) y 6) el crecimiento, para examinar sus efectos sobre el apalancamiento. Los resultados mostraron que estas seis variables independientes 
explicaron el $90 \%$ de la variación en la variable dependiente y, con excepción de la tangibilidad, los resultados fueron altamente significativos.

En otro estudio reciente, Bitjukova, Markovskaya, Bruz y Vititnev (2019) trataron la relación entre el apalancamiento financiero y el valor de una empresa en la industria química; encontraron que la relación entre el apalancamiento financiero y el valor de la empresa fue positiva. Se mostró que las grandes cantidades de deuda que superan el nivel óptimo influyeron en sentido negativo en el valor de mercado de la empresa. También se encontró que la estabilidad financiera incidió en la relación entre el apalancamiento y el valor solo en parte. La salud financiera fortaleció la relación, mientras que el crecimiento tuvo poca o ninguna influencia en ella. La prueba de los patrones de cambio de influencia antes y después de la crisis financiera de 2008 mostró una diferencia significativa únicamente en la relación entre el valor y el apalancamiento financiero al cuadrado.

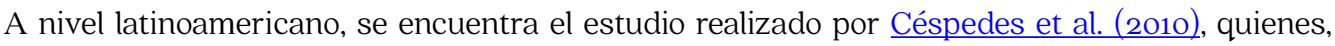
con una muestra de empresas de siete países, mostraron que hay relación entre la concentración de la propiedad y el apalancamiento, a la vez que aportaron evidencia empírica a hallazgos previos de que el tamaño, las oportunidades de crecimiento, la tangibilidad de los activos, la rentabilidad y los impuestos son estadística y económicamente importantes para explicar el apalancamiento en la muestra de empresas latinoamericanas. Hernández et al. (2015) analizaron los determinantes microeconómicos del acceso al financiamiento externo que afectan la estructura de capital de las empresas del sector industrial en México y encontraron que empresas exportadoras y de gran tamaño, al resolver problemas de información asimétrica con sus acreedores, logran continuar financiándose con sus acreedores a pesar de la crisis. Sin embargo, el estudio se concentra en los determinantes microeconómicos principalmente.

En Colombia se han desarrollado estudios enmarcados en la estructura de capital, como el de Pérez (2005), en el que se encontraron similitudes en la estructura de capital de las grandes empresas del sector de alimentos en Colombia, así como también la investigación de Quintero y Rueda (2018), en la que se analizaron los factores en los que se basan los administradores financieros para tomar decisiones sobre el endeudamiento para compañías que cotizan en bolsa. Por su parte, Virgen y Rivera (2012) muestran que en empresas del subsector droguería-cosméticos y hogar-aseo, en Colombia, la rentabilidad y el costo de la deuda son determinantes de la reducción de los niveles de endeudamiento, pese a que las variables influyentes de la estructura financiera de las empresas que conforman un subsector difieren de manera importante del otro subsector.

En este ejercicio de revisión de literatura, el foco estuvo, principalmente, en los estudios que analizan el vínculo entre la estructura de capital y demás variables financieras. Aunque los estudios de estructura de capital se han realizado en múltiples países y en períodos diferentes, los resultados no parecen ser concluyentes, por lo que no se pueden generalizar los resultados para ser aplicados a Colombia, lo que justifica llevar a cabo el presente estudio para la industria química nacional. La Tabla 1 resume los autores, el tamaño de la muestra, el país, el período que comprende el estudio, la variable independiente utilizada en cada uno, el método de estimación y los principales hallazgos. 
Tabla 1. Estudios que evalúan los determinantes de la estructura de capital en el sector químico en el mundo

\begin{tabular}{|c|c|c|c|c|c|c|}
\hline Autores & $\begin{array}{l}\text { Tamaño de } \\
\text { la muestra }\end{array}$ & País & $\begin{array}{l}\text { Período } \\
\text { estudiado }\end{array}$ & $\begin{array}{c}\text { Variable } \\
\text { dependiente }\end{array}$ & Método & Hallazgos \\
\hline$\frac{\text { Cintra et al. }}{\underline{(2012)}}$ & $\begin{array}{l}12 \\
\text { empresas }\end{array}$ & Brasil & $\begin{array}{l}1999- \\
2008\end{array}$ & $\begin{array}{l}\text { Nivel de } \\
\text { endeudamiento }\end{array}$ & $\begin{array}{l}\text { Regresión MCO en } \\
\text { un análisis de datos } \\
\text { de panel. Efectos } \\
\text { fijos y aleatorios. }\end{array}$ & $\begin{array}{l}\text { Las variables tamaño, } \\
\text { la tangibilidad de los } \\
\text { activos, la intangibilidad, } \\
\text { la rentabilidad y el riesgo } \\
\text { fueron importantes } \\
\text { para explicar el nivel de } \\
\text { endeudamiento de las } \\
\text { firmas analizadas. }\end{array}$ \\
\hline $\begin{array}{l}\frac{\text { Camara- }}{\text { Turull et al. }} \\
\underline{(2017)}\end{array}$ & $\begin{array}{l}151 \\
\text { empresas }\end{array}$ & España & $\begin{array}{l}1999- \\
2013\end{array}$ & $\begin{array}{l}\text { Nivel de } \\
\text { endeudamiento }\end{array}$ & $\begin{array}{l}\text { Mapas } \\
\text { autoorganizados } \\
\text { (self-organizing } \\
\text { maps, SOM). }\end{array}$ & $\begin{array}{l}\text { Las compañías se } \\
\text { agruparon en diferentes } \\
\text { grados de cumplimiento } \\
\text { de la teoría del orden } \\
\text { jerárquico (POT). }\end{array}$ \\
\hline$\frac{\frac{\text { Bodla y }}{\text { Singh }}}{\underline{(2017)}}$ & $\begin{array}{l}120 \\
\text { empresas } \\
\text { (60 de } \\
\text { cada una } \\
\text { de las dos } \\
\text { industrias) }\end{array}$ & India & $\begin{array}{l}1999- \\
2015\end{array}$ & $\begin{array}{l}\text { Nivel de } \\
\text { endeudamiento }\end{array}$ & Regresión MCO. & $\begin{array}{l}\text { El costo de la deuda, el } \\
\text { costo del patrimonio, } \\
\text { la liquidez y el servicio } \\
\text { de la deuda resultaron } \\
\text { significativas para } \\
\text { determinar la estructura de } \\
\text { capital. }\end{array}$ \\
\hline $\begin{array}{l}\frac{\text { Zelgalve y }}{\text { Bērzkalne }} \\
\underline{(2011)}\end{array}$ & $\begin{array}{l}10 \\
\text { empresas }\end{array}$ & Letonia & $\begin{array}{l}2000- \\
2008\end{array}$ & $\begin{array}{l}\text { Nivel de } \\
\text { endeudamiento }\end{array}$ & $\begin{array}{l}\text { Métodos de } \\
\text { investigación } \\
\text { cualitativos y } \\
\text { cuantitativos: } \\
\text { análisis } \\
\text { monográfico, } \\
\text { de series de } \\
\text { tiempo, análisis } \\
\text { factorial, regresión } \\
\text { multifactorial y } \\
\text { correlación, } \\
\text { encuesta y el } \\
\text { método gráfico. }\end{array}$ & $\begin{array}{l}\text { El } 80 \% \text { de las empresas } \\
\text { cumplió la teoría del tiempo } \\
\text { del mercado; para un } 10 \% \\
\text { no fue posible establecer } \\
\text { un cumplimiento preciso } \\
\text { debido a la falta de datos; } \\
\text { y en el } 10 \% \text { restante de las } \\
\text { empresas se encontraron } \\
\text { rasgos de la POT. }\end{array}$ \\
\hline $\begin{array}{l}\text { Muhammad } \\
\frac{\text { et al. }}{(2008)}\end{array}$ & $\begin{array}{l}26 \\
\text { empresas }\end{array}$ & Pakistán & 1993-2004 & $\begin{array}{l}\text { Nivel de } \\
\text { endeudamiento }\end{array}$ & $\begin{array}{l}\text { Regresión agrupada } \\
\text { en un análisis de } \\
\text { datos de panel. }\end{array}$ & $\begin{array}{l}\text { Las variables tamaño de la } \\
\text { empresa, la tangibilidad de } \\
\text { los activos, la rentabilidad, } \\
\text { la variación de los ingresos, } \\
\text { el escudo fiscal sin deuda } \\
\text { (NDTS) y el crecimiento } \\
\text { explicaron el } 90 \% \text { de la } \\
\text { variación en la variable } \\
\text { dependiente. }\end{array}$ \\
\hline $\begin{array}{l}\text { Bitjukova } \\
\underline{\text { et al. (2019) }}\end{array}$ & $\begin{array}{l}12945 \\
\text { empresas- } \\
\text { año }\end{array}$ & $\begin{array}{l}\text { No se } \\
\text { especifica }\end{array}$ & $\begin{array}{l}1998- \\
2017\end{array}$ & $\begin{array}{l}\text { Apalancamiento } \\
\text { financiero }\end{array}$ & $\begin{array}{l}\text { Efectos fijos } \\
\text { de panely } \\
\text { estimaciones } \\
\text { agrupadas de } \\
\text { mínimos cuadrados } \\
\text { ordinarios. }\end{array}$ & $\begin{array}{l}\text { La relación entre el } \\
\text { apalancamiento financiero } \\
\text { y el valor es positiva. La } \\
\text { estabilidad financiera } \\
\text { influye en la relación } \\
\text { entre el apalancamiento } \\
\text { y el valor solo en parte. El } \\
\text { crecimiento tiene poca o } \\
\text { ninguna influencia en la } \\
\text { relación. }\end{array}$ \\
\hline
\end{tabular}




\begin{tabular}{|c|c|c|c|c|c|c|}
\hline Autores & $\begin{array}{l}\text { Tamaño de } \\
\text { la muestra }\end{array}$ & País & $\begin{array}{l}\text { Período } \\
\text { estudiado }\end{array}$ & $\begin{array}{c}\text { Variable } \\
\text { dependiente }\end{array}$ & Método & Hallazgos \\
\hline $\begin{array}{l}\text { Céspedes } \\
\text { et al. }(2010)\end{array}$ & $\begin{array}{l}806 \\
\text { empresas }\end{array}$ & $\begin{array}{l}\text { Argentina, } \\
\text { Brasil, } \\
\text { Chile, } \\
\text { Colombia, } \\
\text { México, } \\
\text { Perú, y } \\
\text { Venezuela }\end{array}$ & $\begin{array}{l}1996- \\
2005\end{array}$ & $\begin{array}{l}\text { Nivel de } \\
\text { endeudamiento }\end{array}$ & $\begin{array}{l}\text { Regresión MCO } \\
\text { en un análisis de } \\
\text { datos de panel con } \\
\text { efectos fijos. }\end{array}$ & $\begin{array}{l}\text { El tamaño, las } \\
\text { oportunidades de } \\
\text { crecimiento, la tangibilidad } \\
\text { de los activos, la } \\
\text { rentabilidad y los } \\
\text { impuestos son estadística } \\
\text { y económicamente } \\
\text { importantes para explicar } \\
\text { el apalancamiento en la } \\
\text { muestra de empresas } \\
\text { latinoamericanas. }\end{array}$ \\
\hline$\frac{\text { Hernández }}{\text { et al. }(2015)}$ & $\begin{array}{l}44 \\
\text { empresas }\end{array}$ & México & $\begin{array}{l}2000- \\
2010\end{array}$ & $\begin{array}{l}\text { Apalancamiento } \\
\text { en moneda } \\
\text { extranjera }\end{array}$ & $\begin{array}{l}\text { Regresión de } \\
\text { datos de panel, } \\
\text { por efectos fijos y } \\
\text { aleatorios. }\end{array}$ & $\begin{array}{l}\text { Las empresas exportadoras } \\
\text { y de gran tamaño, al } \\
\text { resolver problemas de } \\
\text { información asimétrica } \\
\text { con sus acreedores, logran } \\
\text { continuar financiándose } \\
\text { con sus acreedores a pesar } \\
\text { de la crisis. }\end{array}$ \\
\hline$\frac{\frac{\text { Virgen }}{\text { y Rivera }}}{\underline{(2012)}}$ & $\begin{array}{l}45 \\
\text { empresas } \\
\text { para el } \\
\text { subsector } \\
\text { droguería- } \\
\text { cosméticos } \\
\text { y } 40 \text { para el } \\
\text { de hogar- } \\
\text { aseo }\end{array}$ & Colombia & $2004-2010$ & $\begin{array}{l}\text { Nivel de } \\
\text { endeudamiento }\end{array}$ & $\begin{array}{l}\text { Modelos estáticos } \\
\text { por mínimos } \\
\text { cuadrados } \\
\text { adrupados (pols), } \\
\text { efectos fijos (fe) y } \\
\text { efectos aleatorios } \\
\text { (re). }\end{array}$ & $\begin{array}{l}\text { La rentabilidad y el costo de } \\
\text { la deuda son determinantes } \\
\text { de la reducción de los } \\
\text { niveles de endeudamiento. }\end{array}$ \\
\hline$\frac{\frac{\text { Quintero }}{\text { y Rueda }}}{\underline{\text { (2018) }}}$ & $\begin{array}{l}42 \\
\text { empresas }\end{array}$ & Colombia & $2010-2017$ & $\begin{array}{l}\text { Tasa de } \\
\text { Variación en } \\
\text { el nivel de } \\
\text { endeudamiento }\end{array}$ & $\begin{array}{l}\text { Regresión de datos } \\
\text { de panel por efectos } \\
\text { fijos. }\end{array}$ & $\begin{array}{l}\text { Las variables } \\
\text { macroeconómicas y } \\
\text { financieras utilizadas para el } \\
\text { modelo son determinantes } \\
\text { al momento en que las } \\
\text { compañías deciden buscar } \\
\text { recursos de financiación o } \\
\text { modificar su estructura de } \\
\text { capital. }\end{array}$ \\
\hline
\end{tabular}

Fuente: elaboración de los autores

\subsection{Análisis de la industria química}

La industria química ha hecho parte integral de la economía global por muchos siglos. En la actualidad, juega un papel crucial en las economías regionales de casi todos los rincones del mundo. Esta industria produce una gran cantidad de productos terminados de consumo general, como fertilizantes, pesticidas y revestimientos para la construcción, y, a su vez, elabora insumos básicos para otras actividades que aseguran los estándares de calidad de vida de los consumidores alrededor del mundo, que se usan, por ejemplo, para el tratamiento de agua potable y la producción de fibras como nilón y poliestireno (Oxford Economics, 2019).

La manufactura del sector es de las más grandes del mundo y demanda aire, agua, gas natural, metales, aceites y diversos minerales, entre otros insumos, para la producción de una variedad de productos químicos que se utilizan en todo el planeta (KenResearch, 2017). Sin embargo, la industria química se enfrenta a una serie de desafíos importantes, desde la reducción de su dependencia de los combustibles fósiles hasta su papel en la lucha contra el cambio climático en general (Bissember, 2017). El mercado químico mundial está segmentado según el tipo de producto y la geografía. De acuerdo con KenResearch (2017), en 2016 se observó que Asia representaba alrededor del 6o \% de la cuota de mercado y se convirtió en la región más grande en el mercado de productos químicos. 
De acuerdo con el informe, la industria química de Asia Pacífico fue la que tuvo la mayor contribución anual al PIB y al empleo. Este mercado ha sido el de más rápido crecimiento debido a los excelentes resultados de China e India. La mitad de las ventas del mercado químico de Asia correspondió a China, Singapur y Japón. Las principales razones del crecimiento en la región asiática han sido el bajo costo de la mano de obra y la concentración de las industrias manufactureras, lo que condujo aún más al dominio en el mercado mundial (KenResearch, 2017).

\section{Metodología}

En esta sección se presenta lo referente a las fuentes de información, el tamaño de la muestra, la medición de las variables y una discusión de las diferentes medidas de las variables.

\subsection{Datos}

El estudio tomó la información financiera de empresas colombianas del sector químico. La información utilizada comprende un período de diez años entre 2009 y 2018. Los datos tienen una frecuencia anual y corresponden a información financiera de las compañías analizadas. La información se obtuvo de la base de datos Emis Benchmark® (s. f.).

Al inicio se contó con datos de diez años de 573 empresas, pero, una vez se eliminaron las compañías con datos faltantes, se seleccionaron 233 firmas para el análisis de datos de panel. En la muestra de las 233 organizaciones ( 2330 datos durante diez años) se aplicaron algoritmos que suprimieron los datos atípicos, lo que redujo el $15 \%$ de los datos y dejó 1980 observaciones para el desarrollo del modelo, lo que implicó que el panel objeto de desarrollo fuese desbalanceado.

\subsection{Variables}

El estudio usó las variables rentabilidad, tangibilidad de activos, crecimiento, tamaño, NDTS, liquidez, escudo fiscal, riesgo y costo de agencia como explicativas del apalancamiento en la estructura financiera.

\subsubsection{Variable dependiente}

Como variable dependiente del estudio se utilizó el apalancamiento (LEVE). El apalancamiento se refiere al porcentaje de activos financiados por deuda. Según Fama y French (2002), la POT y la STT se aplican al valor contable de la deuda. El apalancamiento se calcula como pasivos totales sobre activos totales.

$$
\text { LEVE }=\frac{\text { Pasivos }}{\text { Activos }}
$$

Sin embargo, siguiendo a Shah e Hijazi (2004), se utilizó el valor en libros y no el valor de mercado de la compañía para calcular el apalancamiento. Esto se debe a que el primero no modifica los beneficios del escudo fiscal y porque el valor relevante de la deuda para una empresa en quiebra es el valor en libros de la deuda. 


\subsubsection{Variables independientes}

\section{Rentabilidad (PROF)}

La variable rentabilidad se calculó como utilidad antes de impuestos (UAI) sobre total de activos.

$$
\text { PROF }=\frac{\text { Utilidad antes de impuestos }}{\text { Activos }}
$$

Algunos trabajos, como los de Shah e Hijazi (2004) y Tariq e Hijazi (2006), usan este cálculo de rentabilidad en los estudios de estructura de capital.

Tangibilidad de los activos (TANG)

La variable tangibilidad de los activos se calculó como

$$
T A N G=\frac{\text { Activo fijo }}{\text { Activos }}
$$

Rajan y Zingales (1995) argumentaron que, si las empresas tienen activos tangibles que pueden usarse como garantía para las operaciones financieras, se reducirá el riesgo de que el acreedor sufra los costos de la agencia de la deuda, lo que respaldará una relación positiva entre tangibilidad y endeudamiento. Sin embargo, la POT sostiene, como argumentaron Harris y Raviv (1990), que las compañías con bajos niveles de activos fijos tendrían más problemas de información asimétrica, lo que las llevaría a emitir más deuda, puesto que las emisiones de capital solo serían posibles mediante la subestimación de las acciones. Frank y Goyal (2003) afirmaron que la tangibilidad de los activos sería la variable más importante para probar la POT y encontraron un vínculo positivo en sus pruebas entre la tangibilidad de los activos y el apalancamiento de la firma.

\section{Crecimiento (GROW)}

Diferentes estudios han utilizado diversas formas de medir el crecimiento: como el valor de mercado del patrimonio, el gasto de investigación sobre las ventas totales y el aumento porcentual anual de los activos totales (Titman y Wessels, 1988). En el presente estudio, la variable crecimiento se calculó como la variación porcentual de los activos totales.

$$
\text { GROW }=\frac{\text { Activos }_{t}}{\operatorname{Activos}_{t-1}}-1
$$

Titman y Wessels (1988) enfatizaron que las oportunidades de crecimiento no pueden servir como garantía de la deuda, lo que llevaría a una relación negativa entre las oportunidades de crecimiento y el endeudamiento. Para Fama y French (2002), la POT presenta dos posibles relaciones entre el endeudamiento y las oportunidades de inversión. Según el orden jerárquico modificado, las empresas con altas oportunidades de crecimiento mantendrían bajos sus niveles de endeudamiento, con el fin de preservar su capacidad crediticia para cuando sea necesario, porque esperan una relación negativa entre ambas variables. Por otro lado, el orden jerárquico simple sugiere que este crecimiento requiere inversiones que, por lo general, se llevan a cabo con la nueva deuda, con la confianza en una relación positiva entre las variables. 
El tamaño de la empresa se mide como el logaritmo natural de los activos. Esto con el fin de suavizar la variación durante los períodos considerados.

$$
S I Z E=\operatorname{Ln}(\text { Activos })
$$

Con fundamento en el enfoque de STT, Titman y Wessels (1988) afirmaron que las grandes empresas no consideran los costos directos de bancarrota como una variable activa para decidir el nivel de apalancamiento. Rajan y Zingales (1995) expusieron dos resultados esperados para la relación entre el tamaño de la empresa y el nivel de endeudamiento: primero, las empresas más grandes, al estar más diversificadas, tendrían menos probabilidad de bancarrota, lo que conduciría a una relación positiva entre las dos variables y, segundo, las compañías más grandes tendrían menos asimetría de información entre gerentes e inversionistas; por lo tanto, emitirían más valores sensibles a la información, como acciones, y, en consecuencia, existiría una relación negativa entre las variables. Por su parte, Frank y Goyal (2003) argumentaron que esta relación podría ser negativa debido a que hay menos asimetría de información en las grandes empresas, lo que reduce las posibilidades de subvaloración de las emisiones de capital.

Escudo fiscal no proveniente de deuda (NDTS)

El NDTS se mide como la relación entre la depreciación y los activos totales:

$$
\text { NDTS }=\frac{\text { Depreciación }}{\text { Activos }}
$$

Wald (1999) utilizó la relación entre la depreciación y los activos totales y encontró que el apalancamiento se correlacionó en sentido negativo con el NDTS. Rao, Kumar y Madhavan (2019) encontraron que hay una relación inversa entre el NDTS y el endeudamiento calculado de las cuatro formas siguientes: 1) total de préstamos sobre activos totales, 2) total de pasivos sobre la suma del patrimonio y el pasivo total, 3 ) deuda de corto plazo sobre el total de activos y 4) deuda sobre activos totales.

Liquidez (LIQU)

El cálculo de la liquidez se hace al dividir los activos corrientes entre los pasivos corrientes:

$$
L I Q U=\frac{\text { Activo corriente }}{\text { Pasivo corriente }}
$$

Moosa, Li y Naughton (2011) encontraron que la liquidez se asoció en sentido negativo con el apalancamiento. Así mismo, Mateev, Poutziouris e Ivanov (2013) identificaron que las pymes tenían una mayor proporción de pasivos corrientes en su estructura de capital en comparación con las grandes. Plantearon que en las pymes que mantenían liquidez de corto plazo había correlación positiva con el crecimiento; por tanto, enfrentarían menos restricciones financieras. 


\section{Escudo fiscal (TAXS)}

La variable se calculó como impuestos sobre utilidad bruta:

$$
\text { TAXS }=\frac{\text { Impuestos }}{\text { Utilidad bruta }}
$$

Kumar y Yerramilli (2018) encontraron una relación positiva entre el apalancamiento financiero solo para tasas impositivas marginales bajas. En su modelo, los impuestos también afectaron el valor de la opción real y el riesgo de incumplimiento endógeno de la empresa, por lo que el efecto de los impuestos sobre el apalancamiento financiero no fue tan sencillo.

\section{Riesgo (RISK)}

La variable riesgo se calculó como el porcentaje de cambio de un período a otro de la relación de la utilidad antes de impuestos entre el total de activos:

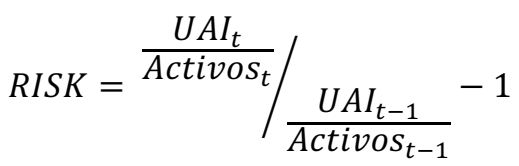

Aunque la teoría sugiere una relación negativa entre el riesgo y el apalancamiento, Burgman $\underline{(1996)}$ encontró que la relación fue positiva para las empresas multinacionales. Mohsin (2016) halló una relación positiva significativa entre el riesgo y la deuda de corto y de largo plazo.

Costo de agencia (COSA)

De acuerdo con Ang, Cole y Lin (2000) y Fleming, Heaney y Mccosker (2005), la variable se calculó como gastos operacionales sobre ventas:

$$
\operatorname{COSA}=\frac{\text { Gastos operacionales }}{\text { Ventas }}
$$

Según Jensen (1986), la deuda es un mecanismo para controlar los comportamientos oportunistas de los gerentes cuando la empresa tiene flujos de efectivo libres y evita que sean explotados, ya sea para su uso personal o con inversiones considerables en proyectos desfavorables.

\subsection{Hipótesis}

La rentabilidad supone un desacuerdo entre teorías de estructura de capital. La del STT afirma que cuanto mayor sea la rentabilidad de la empresa, más razones tendrá para emitir deuda, lo que reduce su carga fiscal. Por otro lado, la POT presupone que mayores ganancias conducen al uso de las ganancias retenidas para suplir el déficit financiero. Por lo tanto, la STT espera una relación positiva entre rentabilidad y apalancamiento, mientras que la POT espera exactamente lo contrario. La primera hipótesis supuso que las empresas con mayor rentabilidad tendrán menor nivel de apalancamiento (Quintero y Rueda, 2018).

Hipótesis 1: existe una relación negativa entre la rentabilidad y el nivel de apalancamiento. 
Las teorías y los estudios empíricos exponen que las empresas con activos que pueden usarse como garantía tienen mayor acceso a financiamiento de bajo costo, debido a que el valor colateral de los activos fijos reduce el riesgo asociado con la deuda. La segunda hipótesis supuso que las compañías con mayor porcentaje de activos fijos tendrán índices de deuda más altos (Cintra et al., 2012).

Hipótesis 2: existe una relación positiva entre el porcentaje de activos fijos y el nivel de apalancamiento.

La STT predice una relación inversa entre el tamaño y la quiebra y, por lo tanto, una relación positiva entre el tamaño y el apalancamiento. La literatura, cuando relaciona la teoría de los costos de bancarrota con el tamaño de la empresa, sugiere que las grandes organizaciones están más diversificadas y tienen un menor riesgo de dificultades financieras, por lo que cuentan con mayor capacidad de financiamiento. Por otro lado, si se toma el tamaño como un proxy para la asimetría de información, las grandes empresas tienden a revelar más información interna debido a que de manera permanente son observadas por analistas del mercado de capitales. Entonces, la asimetría de información entre los expertos y los inversores en el mercado de capitales es menor para las grandes compañías. En consecuencia, la POT predice una relación negativa entre el tamaño y el apalancamiento. La tercera hipótesis consideró una relación negativa entre el tamaño de la empresa y el nivel de apalancamiento (Muhammad et al., 2008).

Hipótesis 3: existe una relación negativa entre el tamaño de la empresa y el nivel de apalancamiento.

La teoría supone que el NDTS se asocia en sentido negativo con el apalancamiento porque el último se considera un sustituto del escudo fiscal que obtiene la compañía al financiarse con deuda. La cuarta hipótesis supuso una relación inversa entre el NDTS y el apalancamiento (Muhammad et al., 2008).

Hipótesis 4: existe una relación negativa entre el NDTS y el apalancamiento.

Las consideraciones sobre la liquidez de la empresa toman en cuenta los activos y los pasivos corrientes. Los activos corrientes incluyen deudores, inventarios, efectivo y todos los demás valores negociables; los pasivos corrientes comprenden acreedores, préstamos bancarios de corto plazo y otras deudas por un período de menos de un año. La quinta hipótesis supone que la liquidez está relacionada en sentido negativo con el apalancamiento (Bodla y Singh, 2017).

Hipótesis 5: existe una relación negativa entre la liquidez y el apalancamiento.

El principal beneficio de la deuda se obtiene de las protecciones fiscales de intereses para la empresa siempre que ella cumpla sus obligaciones. Según la STT, se espera que el efecto del escudo fiscal sobre el endeudamiento sea positivo. La sexta hipótesis estableció que entre mayor sea el escudo fiscal, mayor será el apalancamiento de la empresa (Muhammad et al., 2008).

Hipótesis 6: existe una relación positiva entre el escudo fiscal y el apalancamiento.

La volatilidad que se presenta en las ganancias antes de impuestos y de intereses aumenta el factor de riesgo asociado a las empresas, por lo que los prestamistas exigen primas más altas que hacen que la opción de deuda sea menos atractiva. Esto lleva al planteamiento de la séptima hipótesis: entre mayor sea el riesgo, menor será el apalancamiento de la compañía (Cintra et al., 2012).

Hipótesis 7: existe una relación negativa entre el riesgo y el apalancamiento. 


\subsection{Técnica analítica}

Este estudio utilizó análisis de regresión de panel. Este método facilita el análisis de datos de series transversales y de tiempo y para ello se emplea una regresión agrupada. La ventaja de este modelo es que dispone de mayor cantidad de datos y puede hacer un seguimiento de cada firma. La desventaja de utilizar esta técnica surge porque si todas las cualidades relevantes de la firma no son observables, los errores individuales estarán correlacionados con las observaciones y los mínimos cuadrados ordinarios (MCO) no serán consistentes.

Para solucionar los inconvenientes que se pudieran presentar en el modelo se han propuesto modelos alternativos a la regresión agrupada mediante el anidamiento de los datos: el de efectos fijos y el de efectos aleatorios (Montero, 2011). El modelo de efectos fijos supone que el error puede descomponerse en dos porciones: una parte fija constante para cada individuo $\left(v_{i}\right)$ y otra aleatoria que cumple los requisitos de $\mathrm{MCO}$. El modelo de efectos aleatorios hace consideraciones similares para el comportamiento de los errores, salvo que $v_{i}$ es una variable aleatoria con valor medio de $v_{i} \mathrm{y}$ $\operatorname{varianza} \operatorname{Var}(v i) \neq 0$.

En la muestra inicial se aplicaron algoritmos que detectan y eliminan datos atípicos en la información para lograr un mejor ajuste; algunos algoritmos clásicos los expusieron Zhao, Nasrullah y Li (2019). Con posterioridad, se aplicaron pruebas de especificación para determinar cuál de dichos modelos sería el más apropiado. La prueba de Breusch y Pagan ayudó a determinar si la varianza de $v_{i}$ fue significativamente distinta de cero, con el fin de escoger entre MCO o el modelo anidado. La prueba de Hausman informó acerca de si las estimaciones consistentes y eficientes fueron significativamente diferentes, lo que permitió determinar si se escogía el modelo de efectos fijos o el de efectos aleatorios. El modelo objeto de estimación presenta la siguiente forma:

$$
\begin{aligned}
L_{E V E_{t i}=\beta_{0}}+ & \beta_{1} \text { PROF }_{i t}+\beta_{2} \text { TANG }_{i t}+\beta_{3} \text { GROW }_{i t}+\beta_{4} \operatorname{SIZE}_{i t}+\beta_{5} \text { NDTS }_{i t} \\
& +\beta_{6} \operatorname{LIQU}_{i t}+\beta_{7} \text { TAXS }_{i t}+\beta_{8} \text { RISK }_{i t}+\beta_{9} \operatorname{COSA}_{i t}+u_{i t}
\end{aligned}
$$




\section{Resultados}

Los resultados exponen los hallazgos encontrados después de aplicar las diferentes técnicas analíticas. En la Tabla 2 se muestran las estadísticas descriptivas de las variables utilizadas en el estudio. Como se puede observar en esta tabla (Tabla 2), las empresas que componen la muestra para el estudio presentan un endeudamiento promedio del $50 \%$. En las variables dependientes se observa que la de mayor volatilidad es el riesgo, y la de menor volatilidad es el escudo fiscal.

Tabla 2. Estadísticas descriptivas

\begin{tabular}{|c|c|c|c|c|c|c|}
\hline & & & & Percent & & \\
\hline & Promedio & Mín. & 25 & 50 & 75 & Máx. \\
\hline LEVE & $50 \%$ & $13 \%$ & $36 \%$ & $50 \%$ & $63 \%$ & $130 \%$ \\
\hline PROF & $11 \%$ & $-37 \%$ & $4 \%$ & $8 \%$ & $15 \%$ & $71 \%$ \\
\hline TANG & $19 \%$ & $0 \%$ & $7 \%$ & $16 \%$ & $27 \%$ & $83 \%$ \\
\hline SIZE & 9,80 & 6,30 & 8,50 & 9,48 & 10,95 & 14,63 \\
\hline GROW & $2,2 \%$ & $-2,0 \%$ & $0,9 \%$ & $1,8 \%$ & $3,0 \%$ & $22,0 \%$ \\
\hline NDTS & $2 \%$ & $-2 \%$ & $1 \%$ & $2 \%$ & $3 \%$ & $22 \%$ \\
\hline LIQU & 2,36 & 0,38 & 1,59 & 2,08 & 2,90 & 6,52 \\
\hline TAXS & $7 \%$ & $0 \%$ & $3 \%$ & $6 \%$ & $10 \%$ & $26 \%$ \\
\hline RISK & $(0,00)$ & $(9,68)$ & $(0,34)$ & $(0,04)$ & 0,28 & 7,97 \\
\hline $\cos A$ & $31 \%$ & $-18 \%$ & $20 \%$ & $29 \%$ & $41 \%$ & $76 \%$ \\
\hline
\end{tabular}

Fuente: Elaboración propia

\subsection{Matriz de correlación}

En la Tabla 3 se muestra la matriz de correlación, en la que se aprecia la dureza en la asociación lineal entre todas las variables, con inclusión de la variable de respuesta. Se puede observar, a priori, una alta asociación lineal negativa del apalancamiento (LEVE) con la liquidez (LIQU), lo que demuestra que el resultado matemático hasta el momento es coherente con el postulado teórico. Con respecto a las demás, no hubo evidencia de correlación fuerte entre las variables (Quintero y Rueda, 2018; Bodla y Singh, 2017). 
Tabla 3. Matriz de correlación

\begin{tabular}{lc|c|c|c|c|c|c|c|c|c}
\hline & LEVE & PROF & TANG & SIZE & GROW & NDTS & LIQU & TAXS & RISK & COSA \\
\hline LEVE & 1,00 & $-0,28$ & $-0,11$ & 0,00 & 0,08 & 0,02 & $-0,52$ & $-0,18$ & $-0,01$ & 0,07 \\
\hline PROF & $-0,28$ & 1,00 & $-0,09$ & $-0,05$ & 0,12 & $-0,08$ & 0,18 & 0,34 & 0,02 & $-0,12$ \\
\hline TANG & $-0,11$ & $-0,09$ & 1,00 & 0,04 & $-0,06$ & 0,27 & $-0,11$ & 0,03 & $-0,05$ & $-0,09$ \\
\hline SIZE & 0,00 & $-0,05$ & 0,04 & 1,00 & $-0,05$ & $-0,06$ & 0,15 & 0,08 & 0,01 & $-0,12$ \\
\hline GROW & 0,08 & 0,12 & $-0,06$ & $-0,05$ & 1,00 & $-0,12$ & $-0,05$ & $-0,01$ & 0,01 & $-0,10$ \\
\hline NDTS & 0,02 & $-0,08$ & 0,27 & $-0,06$ & $-0,12$ & 1,00 & $-0,10$ & $-0,02$ & $-0,04$ & 0,10 \\
\hline LIQU & $-0,52$ & 0,18 & $-0,11$ & 0,15 & $-0,05$ & $-0,10$ & 1,00 & 0,07 & 0,00 & $-0,04$ \\
\hline TAXS & $-0,18$ & 0,34 & 0,03 & 0,08 & $-0,01$ & $-0,02$ & 0,07 & 1,00 & 0,00 & 0,21 \\
\hline
\end{tabular}

Fuente: elaboración propia

\subsection{Ajustes al modelo}

Con el fin de validar que el modelo no presente problemas de heterocesaticidad, se realizó la prueba de Breuch y Pagan (1979). Según esta prueba, se presentan la hipótesis nula Ho: los datos son homocedásticos, y la hipótesis alternativa Ha: los datos son heterocedásticos. El multiplicador de Lagrange se usó como estadístico de prueba para validar la hipótesis propuesta.

La Tabla 4 muestra los resultados de la prueba, que arrojó un valor p menor que $1 \%$, de modo que se debe rechazar la hipótesis nula de no heterocedasticidad con un $99 \%$ de confianza. Por ello se concluyó que el modelo que se debe utilizar es el de efectos fijos y efectos aleatorios con datos anidados. La prueba se aplicó sobre la estimación de MCO y el modelo de efectos fijos con un nivel de significancia del $1 \%$. Las estimaciones incluyeron todas las variables regresoras.

Tabla 4. Prueba de Breusch y Pagan

\begin{tabular}{l|l} 
Multiplicador de Lagrange & 111,22527 \\
\hline Valor $\mathbf{p}$ & $4,73755 \mathrm{e}-15$ \\
\hline
\end{tabular}

Fuente: elaboración propia 
La prueba de Hausman intenta probar la hipótesis de que el error específico del grupo no está asociado entre sí; por tanto, el modelo de efectos aleatorios es más adecuado que el de efectos fijos. Así, Ho: efectos aleatorios y Ha: efectos fijos. Se usó el estadístico H para validar la hipótesis propuesta:

$$
H=\left(\beta_{c}-\beta_{e}\right)^{\prime}\left(V_{c}-V_{e}\right)^{-1}\left(\beta_{c}-\beta_{e}\right) \quad H \sim \chi_{n}^{2}
$$

Donde:

$\beta_{c} \quad$ vector de estimaciones del estimador consistente

$\beta_{\boldsymbol{e}} \quad$ vector de estimaciones del estimador eficiente

$V_{c} \quad$ matriz de covarianzas del estimador consistente

$V_{\boldsymbol{e}} \quad$ matriz de covarianzas del estimador eficiente

n $\quad$ grados de libertad (número de variables, incluyendo la constante).

La Tabla 5 muestra los resultados de la prueba. El valor p de la prueba se encontró por debajo del nivel de significancia de $5 \%$, lo que condujo a rechazar la hipótesis nula de que el error no estuvo asociado con efectos aleatorios; por tanto, se concluye que el modelo adecuado es el de efectos fijos. Además, la prueba de Hausman mostró que el $71,74 \%$ de los errores fue explicado por el modelo de efectos fijos, por lo que no se recurrió al de efectos variables.

Tabla 5. Prueba de Hausman

\begin{tabular}{l|l} 
Variable & Valor \\
\hline Estadístico H & $-989,1389$ \\
\hline Valor $P$ & $4,73 e-15$ \\
\hline Efectos fijos & 0,015383 \\
\hline Residuales & 0,006059 \\
\hline Porcentaje debido a los efectos fijos & 0,717430 \\
\hline
\end{tabular}

Fuente: elaboración propia

La prueba de Shapiro y Wilk busca establecer si una muestra de datos proviene de una distribución normal y para ello plantea:

$$
H o: X \sim N\left(\mu, \sigma^{2}\right) \quad H a: X \nsim N\left(\mu, \sigma^{2}\right)
$$

La Tabla 6 muestra los resultados de las pruebas individuales de cada una de las variables. En ella se puede observar que de acuerdo con el valor p para todas las variables, la prueba individual no rechazó la hipótesis nula con un nivel de significancia del $5 \%$; por tanto, se infiere que los datos provienen de una distribución normal. 
Tabla 6. Prueba de Shapiro y Wilk

\begin{tabular}{l|l} 
Variable & Valor $p$ \\
PROF & $3,20204 \mathrm{E}-26$ \\
\hline GROW & $8,48109 \mathrm{E}-25$ \\
\hline SIZE & $2,33723 \mathrm{E}-12$ \\
\hline LIQU & $2,72983 \mathrm{E}-35$ \\
\hline COSA & $5,94853 \mathrm{E}-17$ \\
\hline
\end{tabular}

Fuente: elaboración propia

\section{$4 \cdot 3$ Resultados de la regresión}

Después de haber definido las diferentes hipótesis y las pruebas estadísticas que le dan validez al ejercicio cuantitativo, se revisó la pertinencia de los resultados. Una estimación preliminar de la regresión de un modelo de efectos fijos con todas las variables seleccionadas para el estudio arrojó que las variables tangibilidad, NDTS, escudo fiscal, y riesgo no resultaron significativas. Por tanto, se llevó a cabo una nueva regresión únicamente con las variables rentabilidad operacional, crecimiento, tamaño, liquidez y costos de agencia. La Tabla 7 muestra los resultados de la regresión en la que se encontró un ajuste global (coeficiente de determinación o R2) de o,3299. Así mismo, se observa que el $32 \%$ del error total del modelo fue explicado por las variables seleccionadas y el $68 \%$ restante por factores aleatorios. La significancia individual de las variables mostró, a través de la revisión de su valor $\mathrm{p}$, que todas las variables incluidas aportaron a la explicación global del modelo ajustado.

Tabla 7. Regresión con modelo de efectos fijos

\begin{tabular}{|c|c|c|c|c|c|c|}
\hline \multicolumn{2}{|c|}{ Variable dependiente } & LEVE & \multicolumn{3}{|l|}{ R cuadrado } & 0,3299 \\
\hline \multicolumn{2}{|l|}{ Estimador } & PanelOLS & \multicolumn{3}{|c|}{ R cuadrado (between) } & 0,2752 \\
\hline \multicolumn{2}{|c|}{ N. ${ }^{\circ}$ de observaciones } & 1980 & \multicolumn{3}{|c|}{ R cuadrado (within) } & 0,3299 \\
\hline \multicolumn{2}{|l|}{ Entidades } & 233 & \multicolumn{3}{|l|}{ Distribución } & $F(14,1734)$ \\
\hline \multicolumn{2}{|l|}{ Períodos } & 10 & \multicolumn{3}{|l|}{ Estadístico F } & 60,979 \\
\hline \multicolumn{2}{|c|}{ Promedio de observaciones } & 8,4979 & \multicolumn{3}{|l|}{ Valorp } & 0 \\
\hline \multicolumn{7}{|c|}{ Estimación de parámetros } \\
\hline Variable & Parámetro & Error estándar & Estadístico t & Valorp & $\begin{array}{l}\text { Intervalo } \\
\text { inferior }\end{array}$ & $\begin{array}{l}\text { Intervalo } \\
\text { superior }\end{array}$ \\
\hline PROF & $-0,3485$ & 0,0357 & $-9,7531$ & 0,0000 & $-0,4185$ & $-0,2784$ \\
\hline GROW & 0,0605 & 0,0145 & 4,1831 & 0,0000 & 0,0322 & 0,0889 \\
\hline SIZE & $-0,0260$ & 0,0092 & 2,8220 & 0,0048 & $-0,0441$ & $-0,0079$ \\
\hline LIQU & $-0,0849$ & 0,0037 & $-22,762$ & 0,0000 & $-0,0922$ & $-0,0776$ \\
\hline $\cos A$ & $-0,1376$ & 0,0489 & $-2,8133$ & 0,0050 & $-0,2335$ & $-0,0417$ \\
\hline
\end{tabular}

Fuente: elaboración propia 
De acuerdo con los resultados obtenidos en el ejercicio de regresión, el nivel de apalancamiento fue explicado en un $32 \%$ por cinco variables: 1) rentabilidad, 2) crecimiento, 3) tamaño, 4) liquidez y 5) costos de agencia. Los resultados señalan una asociación negativa entre la rentabilidad y el nivel de apalancamiento, lo cual soporta la primera hipótesis de este estudio. La rentabilidad fue la variable regresora económicamente más significativa porque presentó el coeficiente más alto. Por tanto, esta evidencia sugiere que las empresas preferirán financiarse con activos propios y pagar la deuda una vez se generen los recursos para ello, lo que concuerda con lo sugerido por la POT.

En cuanto a la hipótesis 2, la evidencia empírica no mostró una relación estadísticamente significativa entre la variable tangibilidad y el nivel de apalancamiento; por tanto, no es posible corroborar este planteamiento. Este resultado contrasta con los hallazgos para empresas latinoamericanas realizado por Céspedes et al. (2010), quienes encuentran significativa esta variable para explicar el apalancamiento de las empresas utilizadas en la muestra analizada.

En el caso de la variable tamaño, los resultados señalan una asociación negativa con el nivel de apalancamiento, por lo que se corrobora el planteamiento de la hipótesis 3, ya que la evidencia empírica muestra que no existe una relación positiva entre el tamaño de la empresa y el nivel de apalancamiento (Muhammad et al., 2008). Este resultado sugiere que es de esperarse que las empresas de mayor tamaño preferirán financiar nuevos proyectos con recursos propios para así desestimar el riesgo asociado con la nueva deuda. Este resultado es coherente con lo supuesto por la POT, como lo explicaron Rajan y Zingales (1995).

En el caso de la variable NDTS, se encontró que no presentó una significancia estadística como determinante en la estructura de capital de las empresas del sector estudiado, en consecuencia, no se corrobora la hipótesis 4 del estudio. Este resultado contrasta con los hallazgos del estudio realizado por Muhammad et al. (2008), quienes encontraron estadística y económicamente significativa esta variable para las empresas analizadas en Pakistán. Por su parte, la variable liquidez presentó una relación negativa con el apalancamiento. Este resultado fue coherente con los resultados expuestos por Moosa et al. (2011), lo que señala que las empresas utilizan los excedentes de liquidez para atender el servicio de la deuda. Este resultado no contradijo lo expuesto por la POT y corrobora la hipótesis 5 del estudio.

En cuanto a la relación entre el escudo fiscal y el apalancamiento, los resultados mostraron que no existe una relación estadísticamente significativa y se evidencia que los flujos percibidos por los escudos fiscales no aportaron a la ecuación de apalancamiento; por tanto, no es posible corroborar la hipótesis 6. Así mismo, tampoco es posible corroborar la hipótesis 7, pues tampoco se encontró una relación estadísticamente significativa entre el riesgo y el apalancamiento, por lo que el riesgo asociado con el flujo de ingresos no fue determinante para la definición de la estructura de capital de las empresas de la muestra de estudio. Esto difiere a lo reportado por Cintra et al. (2012), quienes encontraron significativa esta variable para el caso de las empresas analizadas en Brasil.

Por último, los costos de agencia presentaron una relación negativa con el endeudamiento. Se considera que, ante costos de agencia altos, los gerentes preferirían mantener bajos los niveles de deuda. Esto se consideró coherente si se quiere desestimar el riesgo asociado con la volatilidad de los ingresos. Este resultado refuerza lo expuesto por la POT. En general, los resultados señalan que la POT se ajustó en mayor medida a la estructura de capital de la industria química colombiana. La Tabla 8 presenta 
una comparación entre los distintos planteamientos teóricos de cada una de las variables explicativas respecto al nivel de apalancamiento.

Tabla 8. Comparación de resultados de relaciones entre variables regresoras y predictoras

\begin{tabular}{|c|c|c|c|}
\hline Variable & Resultado teórico & Matriz de correlación & Beta del modelo \\
\hline Rentabilidad (PROF) & Negativa & Negativa & Negativa \\
\hline Tangibilidad (TANG) & Positiva & Negativa & \\
\hline Crecimiento (GROW) & Positiva & & Positiva \\
\hline Tamaño (SIZE) & Positiva & & Negativa \\
\hline Escudo fiscal no proveniente de deuda (NDTS) & Negativa & & \\
\hline Liquidez (LIQU) & Negativa & Negativa & Negativa \\
\hline Escudo fiscal (TAXS) & Positiva & Negativa & \\
\hline Riesogo (RISK) & Negativa & & \\
\hline Costo de agencia (COSA) & Positiva & & Negativa \\
\hline
\end{tabular}

Fuente: elaboración propia

\section{Conclusiones y recomendaciones}

La teoría de estructura de capital ha sido objeto de estudios teóricos y empíricos debido a su relevancia en la sostenibilidad futura de las compañías y al alto grado de atención que recibe por parte de la gerencia. Por esta razón, es importante conocer los determinantes de la estructura de capital que afectan la industria química en Colombia, según los diferentes postulados encontrados. Al hacer la revisión de literatura pertinente para el estudio, se encontraron pocas investigaciones que permitieran conocer cuáles fueron los factores que incidieron en el nivel de apalancamiento de las empresas de la industria química colombiana, por lo que el presente estudio representa una primera aproximación al tema.

Después de haber aplicado la técnica analítica sobre los datos de panel recopilados, se encontró que las variables rentabilidad, crecimiento, tamaño, liquidez y costos de agencia están en la capacidad de explicar el $32 \%$ del nivel de apalancamiento de las empresas de la muestra. Las variables anteriores presentan consistencia con la POT y dan pruebas de la preferencia de los gerentes por la financiación con recursos propios; sin embargo, las teorías de la estructura de capital han sido desarrolladas para explicar fenómenos relacionados con el apalancamiento de empresas listadas en bolsa, mientras que en este estudio se incluyen empresas no listadas. Dado que para estas últimas es mucho más difícil y costoso emitir acciones que deuda (más allá del efecto que pueda tener la asimetría de información), las predicciones de la POT se ven favorecidas en comparación con aquellas predicciones de la STT.

De lo anterior se pueden inferir dos conclusiones importantes. La primera, que el crecimiento de la industria estaría limitado por la capacidad de las empresas para generar recursos propios. La segunda, que en un sector que presenta alto crecimiento, como el estudiado, las compañías necesitan invertir los recursos generados en su propio crecimiento para no abrir posibilidades de entrada a nuevos competidores. Esto último deriva en que las firmas del sector no deben priorizar la entrega de dividendos a los accionistas, a menos que se dé una contracción de la demanda agregada de la industria química.

Así mismo, los resultados encontrados en este estudio tienen implicaciones tanto académicas como prácticas. Por un lado, contribuyen al cuerpo teórico de las finanzas corporativas, particularmente 
en lo referente al estudio de las teorías de estructura de capital en un sector poco estudiado y de alta importancia económica en el mundo. Por tanto, los resultados aportan a la discusión teórica de los determinantes de la estructura de capital en países emergentes caracterizados por entornos de información asimétrica. Por otro lado, sirven como elemento para apoyar la toma de decisiones de los gerentes de empresas del sector al considerar las variables clave del nivel de endeudamiento, así como la forma óptima de administrar los recursos generados por las compañías. Por ello es importante que los gerentes de las empresas de la industria química en Colombia revisen de forma permanente indicadores de rentabilidad, de tamaño o participación de mercado, la tasa de crecimiento de sus activos, la liquidez y los costos asociados con su gestión, pues son variables determinantes para la industria química en las decisiones de financiación. En consecuencia, el comportamiento de estos indicadores mostrará en qué medida la empresa tiene o no capacidad para incurrir en un nivel de financiación óptima y poder aprovechar las oportunidades que se presenten en los mercados de deuda.

Finalmente, para futuros trabajos se recomienda segmentar las empresas que se incluirían en el estudio mediante el uso de la Clasificación Internacional Industrial Uniforme (CIIU). Algunos académicos considerarán que es conveniente analizar el efecto de los ciclos económicos, puesto que se está trabajando con datos de panel. Así mismo, se considera conveniente analizar nuevas técnicas analíticas que permitan lograr, a través de la interacción de las variables, una mayor explicación del modelo. 


\section{Referencias}

Ang, J. S., Cole, R. A. y Lin I. W. (2000). Agency costs and ownership structure. Journal of Finance, 55, 81-106.

Aristizábal Otálvaro, M. C. (2018, 28 de junio). Sector químico. En Estudio sobre bioeconomía. Como fuente de nuevas industrias basadas en el capital natural de Colombia. Fase 2 (pp. 69-74). Medellín: Universidad EAFIT, Biointropic y SILO.

Breuch y Pagan (1979) Breusch, T. S., \& Pagan, A. R. (1979). A simple test for heteroscedasticity and random coefficient variation. Econometrica: Journal of the econometric society, 1287-1294.

Bissember, A. (2017, 26 de marzo). Green chemistry is key to reducing waste and improving sustainability. The Conversation. https://theconversation.com/green-chemistry-is-key-to-reducing-waste-andimproving-sustainability-70740

Bitjukova, A., Markovskaya, E., Bruz, V. y Vititnev, S. (2019). The influence of capital structure on the value of public companies in chemical industry. En E3S Web of Conferences, 135, Innovative Technologies in Environmental Science and Education. https://www.e3s-conferences.org/articles/e3sconf/ abs/2019/61/e3sconf_itese18_04059/e3sconf_itese18_04059.html

Bodla, B. S. y Singh, J. (2017). Determinants of capital structure in India: A study of pharmaceutical and chemical industries. https://www.researchgate.net/publication/321226105_DETERMINANTS_ OF_CAPITAL_STRUCTURE_IN_INDIA_A_STUDY_OF_PHARMACEUTICAL_AND_CHEMICAL_ INDUSTRIES

Burgman, T. A. (1996). An empirical examination of multinational corporate capital structure. Journal of International Business Studies, 27(3), 553-570. https://www.jstor.org/stable/155438?seq=1

Camara-Turull, X., Fernández-Izquierdo, M. Á. y Sorrosal-Forradellas, M. T. (2017). Analysing capital structure of Spanish chemical companies using self-organizing maps. Kybernetes, 46(6), 947-965. http://doi. org/10.1108/K-05-2016-0112

Céspedes, J., González, M. y Molina, C. A. (2010). Ownership and capital structure in Latin America. Journal of Business Research, 63(3), 248-254.

Cintra, N. B., Selan, B., Albanez, T. y Do Valle, M. R. (2012). Estudo comparativo da estrutura de capital e das características de financiamento das empresas do setor químico brasileiro. Enfoque: Reflexão Contábil, 31(2), 75-88.

Durand, D. (1952). Cost of debt and equity funds for business: trends and problems of measurement. En Conference on Research in Business Finance (pp. 215-262). National Bureau of Economic Research. https://www.nber.org/chapters/c4790.pdf

Emis (s. f.). Emis banchmark. Emis. https://www.emis.com/benchmark

Fama, E. F., \& French, K. R. (2002). Testing trade-off and pecking order predictions about dividends and debt. The review of financial studies, 15(1), 1-33.

Fleming, G., Heaney, R. y Mccosker, R. (2005). Agency costs and ownership structure in Australia. PacificBasin Finance Journal, 13(1), 29-52.

Frank y Goyal (2003) Frank, M. Z., \& Goyal, V. K. (2003). Testing the pecking order theory of capital structure. Journal of financial economics, 67(2), 217-248.

Harris, M., \& Raviv, A. (1990). Capital structure and the informational role of debt. The Journal of Finance, 45(2), 321-349.

Hernández C. G., Ríos, H. y Garrido, C. (2015). Determinantes microeconómicos del acceso al financiamiento externo de la estructura de capital de empresas del sector industrial. Contaduría y administración, 60(2), 326-345.

Jensen, M. C. (1986). Agency costs of free cash flow, corporate finance, and takeovers. The American Economic Review, 76(2), 323-329.

Jensen, M. C. y Meckling, W. H. (1976). Theory of the firm: Managerial behavioir, agency cost and ownership structure. Journal of Financial Economics, 305-360. https://www2.bc.edu/thomaschemmanur/phdfincorp/MF891\%20papers/Jensen\%20and\%20Meckling\%201976.pdf 
KenResearch (2017, 18 de septiembre). Global chemical industry growth market report. KenResearch. https://www.kenresearch.com/blog/2017/09/global-chemical-industry-growth-market-report-kenresearch/

Kumar, P. y Yerramilli, V. (2018). Optimal capital structure and investment with real options and endogenous debt costs. The Review of Financial Studies, 31, 3452-3490. https://doi.org/10.1093/rfs/hhx093

Mateev, M., Poutziouris, P. y Ivanov, K. (2013). On the determinants of SME capital structure in Central and Eastern Europe: A dynamic panel analysis. Research in International Business and Finance, 27, 28-51.

Modigliani, F. y Miller, M. H. (1958). The cost of capital, corporation finance and the theory of investment. The American Economic Review, 48(3), 261-297. http://www.jstor.org/stable/1809766

Modigliani, F. y Miller, M. H. (1963). Corporate income taxes and the cost of capital: A correction. American Economic Association, 433-443. https://www.jstor.org/stable/1809167?seq=1

Mohsin, A. (2016). Capital structure determinants for large listed Norwegian and foreign public firms. Oslo and Akershus University College of Applied Sciences.

Montes-Valencia, N. (2015). La industria química: importancia y retos. Lámpsakos, (14), 72-85. http://www. funlam.edu.co/revistas/index.php/lampsakos/article/viewFile/1562/1430

Montero. R (2011). Efectos fijos o aleatorios: test de especificación (documentos de trabajo en Economía Aplicada). Universidad de Granada. España.

Moosa, I., Li, L. y Naughton, T. (2011). Robust and fragile firm-specific determinants of the capital structure of Chinese firms. Applied Financial Economics, 21, 1331-1343. https://doi.org/10.1080/09603107.2011. 570714

Muhammad, R., Asif, I. y Muhammad, A. (2008). The determinants of capital structure of the chemical industry in Pakistan. The Lahore Journal of Economics, 13, 139-158. https://www.researchgate.net/ publication/228319848_The_Determinants_of_Capital_Structure_of_the_Chemical_Industry_in_ Pakistan

Myers, S. C. (1977). Determinants of corporate borrowing. Journal of Financial Economics, 5(2), 147-175. https://doi.org/10.1016/0304-405X(77)90015-0

Myers, S. C. (1984). The capital structure puzzle. The Journal of Finance, 39(3), 575-592. http://citeseerx.ist. psu.edu/viewdoc/download?doi=10.1.1.472.3863\&rep=rep1\&type=pdf

Myers, S. C. y Majluf, N. S. (1984). Corporate financing and investment decisions when firms have information that investors do not have. Journal of Financial Economics, 187-221. https://www.sciencedirect.com/ science/article/abs/pii/0304405X84900230

Oxford Economics (2019). The global chemical industry: catalyzing growth and addressing our world's sustainability challenges. ICCA. https://www.icca-chem.org/wp-content/uploads/2019/03/ ICCA_EconomicAnalysis_Report_030819.pdf

Pérez, A. M. (2005). Análisis de la estructura de capital de las grandes empresas del sector de alimentos en Colombia (1999-2003) (trabajo de grado en Economía). Universidad EAFIT. https://repository.eafit. edu.co/bitstream/handle/10784/506/AnaMaria_PerezRave_2005_.pdf?sequence=1\&isAllowed=y

Quintero, J, y Rueda, F. (2018). Decisión de apalancamiento relacionado con los impactos económicos empresariales - Estructura de capital de las compañías que cotizan en bolsa (trabajo de grado de maestría). Universidad EAFIT. Bogotá. https://repository.eafit.edu.co/bitstream/ handle/10784/13504/Fanny_RuedaBeltran_JulianAndres_QuinteroQuintero_2018. pdf?sequence=2\&isAllowed=y

Rajan, R. G. y Zingales, L. (1995). What do we know about capital structure? Some evidence from international data. The Journal of Finance, 50(5), 1421-1460. https://doi.org/10.1111/j.1540-6261.1995. tb05184.x

Rao, P., Kumar, S. y Madhavan, V. (2019). A study on factors driving the capital structure decisions of small and medium enterprises (SMEs) in India. IIMB Management Review, 31, 37-50.

Ross, S. A. (1977). The determination of financial structure: The incentive-signalling approach. The Bell Journal of Economics, 8(1), 23-40. https://www.jstor.org/stable/3003485?seq=1 
Shah, A. y Hijazi, S. (2004). The determinants of capital structure of stock exchange-listed non-financial firms in Pakistan. The Pakistan Development Review, 43(4), 605-618. http://www.doi.org/10.30541/ v43i4llpp.605-618

Spence, M. (1973). Job market signalling. The Quarterly Journal of Economics, 87(3), 355-374. https://www.jstor.org/stable/1882010?seq=1

Tariq, B. Y. y Hijazi, S. T. (2006). Determinants of capital structure: A case for Pakistani cement industry.

The Lahore Journal of Economics, 11(1), 63-80. https://papers.ssrn.com/sol3/papers.cfm?abstract_ id=892157

Titman, S. y Wessels, R. (1988). The determinants of capital structure choice. The Journal of Finance, 43, 1-19. https://doi.org/10.1111/j.1540-6261.1988.tb02585.x

Virgen, V. y Rivera, J. A. (2012). Financial conditions affecting the financial structure of the cosmetics and household product industries in Colombia. Cuadernos de Administración, 25(44), 175-191.

Wald, I. K. (1999). How firms' characteristics affect capital structure: an international comparison. Journal of Financial Research, 22(2), 161-187. https://doi.org/10.1111/j.1475-6803.1999.tb00721.x

Warner, J. B. (1977). Bankruptcy costs: some evidence. The Journal of Finance, 32(2), 337-347. https://www. jstor.org/stable/2326766?seq=1

Zelgalve, E. y Bērzkalne, I. (2011). Capital raising problems in chemical enterprises: A case of Latvia. Taikomoji Ekonomika, 5(1), 115-129. https://www.vdu.lt/cris/handle/20.500.12259/1327?locale-attribute=en

Zhao, Y., Nasrullah, Z. y Li, Z. (2019). PyOD: A Python toolbox for scalable outlier detection. Journal of Machine Learning Research, 20,1-7. http://www.jmlr.org/papers/volume20/19-011/19-011.pdf 
Anexo. Modelo de efectos fijos con todas las variables (estimación preliminar)

\begin{tabular}{ll|ll}
\hline Variable dependiente & LEVE & R cuadrado & 0,3322 \\
Estimador & PanelOLS & R cuadrado (between) & 0,2305 \\
N.o de observaciones & 1980 & R cuadrado (within) & 0,3322 \\
Entidades & 233 & Distribución & F(18,1730) \\
Periodos & 10 & Estadístico F & 47,822 \\
Promedio de observaciones & 8,4979 & Valor p & 0
\end{tabular}

Estimación de parámetros

\begin{tabular}{|c|c|c|c|c|c|c|}
\hline Variable & Parámetro & Error estándar & Estadístico t & Valor p & $\begin{array}{l}\text { Intervalo } \\
\text { inferior }\end{array}$ & $\begin{array}{l}\text { Intervalo } \\
\text { superior }\end{array}$ \\
\hline PROF & $-0,3923$ & 0,0438 & $-8,9545$ & 0,0000 & $-0,4783$ & $-0,3064$ \\
\hline TANG & 0,0420 & 0,0250 & 1,6792 & 0,0933 & $-0,0071$ & 0,0910 \\
\hline GROW & 0,0650 & 0,0146 & 4,4373 & 0,0000 & 0,0362 & 0,0937 \\
\hline SIZE & $-0,0287$ & 0,0093 & $-3,0888$ & 0,0020 & $-0,0470$ & $-0,0105$ \\
\hline NDTS & 0,0344 & 0,2056 & 0,1676 & 0,8669 & $-0,3688$ & 0,4377 \\
\hline LIQU & $-0,0841$ & 0,0038 & $-22,357$ & 0,0000 & $-0,0915$ & $-0,0767$ \\
\hline TAXS & 0,1116 & 0,0677 & 1,7947 & 0,0729 & $-0,0113$ & 0,2544 \\
\hline RISK & $-2,892 \mathrm{E}-6$ & 1,191E-5 & $-0,2415$ & 0,8092 & $-2,639 E-5$ & 2,06E-5 \\
\hline $\operatorname{COSA}$ & $-0,1278$ & 0,0498 & $-2,5632$ & 0,0105 & $-0,2255$ & $-0,0300$ \\
\hline
\end{tabular}

Fuente: elaboración propia 